\title{
Evaluation of Hemoglobin A1c Levels in Endometrial Cancer Patients: a Retrospective Study in Turkey
}

\author{
Erbil Karaman ${ }^{1 *}$, Yasemin Karaman², Ceyhun Numanoglư ${ }^{3}$, Hasan Cemal Ark ${ }^{4}$
}

\begin{abstract}
Background: Hemoglobin $\mathrm{A1}(\mathrm{HgA1c})$ is a marker of poor gylcemic control and elevation $\mathrm{HgA1c}$ is associated with increased risk of many cancers. We aimed to determine the $\mathrm{HgAlc}$ levels in endometrial cancer cases and any relationship with stage and grade of disease. Materials and Methods: A retrospective data review was performed between June 2011 and October 2012 at a tertiary referral center in Turkey. The study included 35 surgically staged endometrial cancer patients and 40 healthy controls. Preoperative $\mathrm{HgA1c}$ levels drawn within 3 months before surgery were compared. Also the relationships between $\mathrm{HgA1c}$ levels and stage, grade and hystologic type of cancer cases were evaluated. Results: The mean $\mathrm{HgA1c}$ levels were statistically significantly higher at $6.19 \pm 1.44$ in endometrial cancer cases than the $5.61 \pm 0.58$ in controls $(p=0.027)$. With endometrial cancer cases, the mean $\mathrm{HgA1c}$ level was found to be $6.62 \pm 1.40$ for stage I and $6.88 \pm 1.15$ for stages II-IV ( $p=0.07$ ). The figures were $6.74 \pm 1.65$ for endometrioid and $6.63 \pm 1.41$ for non-endometrioid type tumors $(p=0.56)$. Mean HgA1c levels of 6.72 \pm 1.14 for grade 1 and $6.62 \pm 1.42$ for grade $2-3$ were observed $(p=0.57)$. Conclusions: $\mathrm{HgA1c}$ levels in endometrial cancer patients were statistically higher than healthy controls. However, HgA1c did not show any significant correlation with stage, grade and histologic type in endometrial cancer cases.
\end{abstract}

Keywords: Hemoglobin A1c(HgA1c) - endometrial cancer - tumor stage - grade - histological type

Asian Pac J Cancer Prev, 16 (5), 1817-1820

\section{Introduction}

Endometrial cancer, as known, is the most commonly seen gynecologic cancer in developed countries (Binesh et al., 2014). Common estrogen dependent type I and rarer estrogen independent type II are distinguished. It has been reported many risk factors for developing endometrial cancer. Many of these factors are based on the increase of unbalanced estrogen stimulation on endometrium. So it has identifiable risk factors such as hypertension, obesity, diabetes, nulliparity and anovulation (Tangjitgamol et al., 2010).

Although the diabetes mellitus is an independent risk factor for developing of endometrial cancer, it is hypothesized that hyperinsulinemia and impaired glucose metabolism increase the risk of many cancers, including those of the breast, genitourinary and gastrointestinal (Bokham, 1983; Rinaldi et al., 2008). It is reported that the impaired glucose metabolism as measured by $\mathrm{HgA} 1 \mathrm{c}$ without coexistent diabetes disease is seen more in endometrial cancer patients than healthy non-malignant groups (Mahboubi, 1982; Lawrence, 1987). The exact mechanism of diabetes and increased risk of cancer has not yet been explained and remains unclear but it hypothesized that glucose through its action on production of insulin and insulin-like growth factor (IGF)-I may enhance tumor development by stimulating cell proliferation and by inhibiting apoptosis (Kaaks, 2001; LeRoith, 2003). Glycosylated hemoglobin ( $\mathrm{HgAlc})$ is used as a marker for glycemic status of patients and it is often favored over blood glucose level because of to assess glycemic control over a longer period of time. $\mathrm{HgAlc}$ reflects average glucose level over the past three months (Rohlfing et al., 2000).

In a study on colorectal cancer patients, poor glycemic control has been shown to be related to poor overall prognosis. Patients with a measured $\mathrm{HgAlc}>7.5 \%$ had a significantly more aggressive clinical course than those with improved glycemic control (Siddiqui et al., 2008). Also it is reported that diabetes is a independent risk factor for endometrial cancer and has been associated with a poorer survival independent of tumor stage or grade (Folsom et al., 2004). However, marker for impaired glucose control including $\mathrm{HgAlc}$ was not analysed in that study. Also in another study, the elevated markers of poor glycemic control (HgA1c and fasting glucose levels) in patients surgically staged for type I endometrial cancer were evaluated and investigated that whether it is related to a higher stage or higher grade at the time of diagnosis. And it is found that elevated preoperative $\mathrm{HgAlc}$ has a

${ }^{1}$ Department of Obstetrics and Gynecology, Yüzüncüyll University, ${ }^{2}$ Department of Gynecology, Private Lokman Hekim Hayat Hospital, Van, ${ }^{3}$ Department of Gynecological Oncology ${ }^{4}$ Department of Gynecology, Kanuni Sultan Süleyman Research and Teaching Hospital, Istanbul, Turkey*For correspondence: erbil84@gmail.com 
trend toward a higher stage at the time of diagnosis in that study (Stevens et al., 2012). Here, in this study we aimed to evaluate the $\mathrm{HgA} 1 \mathrm{c}$ levels in endometrial cancer cases and sought to determine its relationships with stage and grade of the disease.

\section{Materials and Methods}

A retrospective chart review was performed from June 2011 to October 2012 in gynecologic oncology unit of Kanuni Sultan Süleyman Research and Teaching hospital, İstanbul, Turkey. The institutional review ethical board approved the study. 35 patients operated in gynecologic oncology unit and surgically staged for endometrial cancer were included in study group and 40 patients who were admitted to gynecology clinic for abnormal menstruel bleeding and underwent endometrial biopsy which is revealed benign pathology without hyperplasia or malignancy served as healthy control group. Inclusion criteria in our study group included a hemoglobin A1c performed within 3 months of surgery and surgical staging, including a minimum of hysterectomy and bilateral salpingoopherectomy. Also $5 \mathrm{ml}$ venous blood was drawn from healthy control group prior to the procedure of endometrial biopsy for measurement of $\mathrm{HgA} 1 \mathrm{c}$ levels. The staging was recorded based on the FIGO 2009 revised staging for uterine corpus cancer. Standart FIGO pathological criteria were used to evaluate tumor grade.

The cases with genital system infections, pozitive serum pregnancy test, patients taking hormonal therapy (oral contraception or hormone replacement therapy), patients with systemic disease (other than diabetes or hypertension) and patients using intrauterine device were excluded from study. Demographic characteristics such as including age, menapousal status, diabetes, hypertension, weight, height and body-mass index were determined through review of medical records and tumor registry data. $\mathrm{HgA1c}$ concentrations were measured by method of high performance liquid chromotography using TOSOH HLC 723 G8 analyser equipment (Tosoh bioscience, Japan). The variables and $\mathrm{HgA} 1 \mathrm{c}$ levels were compared between two groups.

Statistical analysis was performed with SPSS statistical package version 20.0 using the t test, Kruskall-Wallis test and chi-square test. For descriptive statistics, the means, standard deviations, and frequencies were calculated. P-values less than 0.05 were considered statistically significant.

\section{Results}

The mean age of endometrial cancer group was $56.80 \pm 10.19$ (range 35-79) which is significantly higher than in control group with mean age of $48.90 \pm 5.79$ (range 39-58) $(\mathrm{p}<0.05)$. The mean parity and gravidity did not show any significant difference between two groups( $\mathrm{p}=0.085, \mathrm{p}=0.384$, respectively). 25 patients in cancer group and 20 patients in control group was postmenapousal and this was not statistically different. There was 11 patients with diagnosis of diabetes disease in cancer group and 9 patients in control group, this was not statistically different $(\mathrm{p}=0.498)$. Also patient with diagnosis of hypertension did not show any significant difference between groups. The mean endometrial thickness in cancer group was significantly higher than control group $(19.20 \pm 10.24$ vs $12.11 \pm 4.25, \mathrm{p}=0.001)$. The demographic data regarding two groups are shown in Table 1 . When analysing the endometrial cancer hystologic types, 25 patients out of 35 cases were endometrioid type, 10 patients were non-endometrioid type. The stage distribution favored early-stage disease, 20 patients surgically staged as stage 1,10 as stage 2,4 as stage 3 and 1 as stage 4 . In terms of tumor grade, $46.2 \%$ had grade 1 tumor and $53.8 \%$ had grade 2-3 tumor.

The overall average $\mathrm{HgA} 1 \mathrm{c}$ was $6.19 \pm 1.44$ in endometrial cancer group and 5.61 \pm 0.58 in control cases which is statistically significantly different $(\mathrm{p}=0.027)$. There was no statistical difference between $\mathrm{HgAlc}$ in early-stage versus later-stage cancers $(\mathrm{p}=0.07)$. Like this, There was no statistical difference between $\mathrm{HgAl}$ c in low-grade versus high grade tumors $(\mathrm{p}=0.57)$. As shown in Table 2, with regards to hystologic types of endometrial cancer, there was no significant diference between $\mathrm{HgA1c}$

Table 2. The HgA1c vs Grade, Stage and Hystologic Type of Cancer Cases

\begin{tabular}{lcc}
\hline & HgA1c & p value \\
\hline Stage & & \\
$\quad$ Stage I (n=20) (mean, SD) & $6.62 \pm 1.40$ & 0.07 \\
$\quad$ Stage II-IV (n=15) (mean, SD) & $6.88 \pm 1.15$ & \\
Grade & & \\
$\quad$ Grade 1(mean, SD) & $6.72 \pm 1.14$ & 0.57 \\
$\quad$ Grade 2-3(mean, SD) & $6.62 \pm 1.42$ & \\
Hystologic type & & \\
$\quad$ Endometrioid (mean, SD) & $6.74 \pm 1.65$ & 0.56 \\
$\quad$ Non-endometrioid(mean, SD) & $6.63 \pm 1.41$ & \\
\hline
\end{tabular}

Table 1. Demographic data and HgA1c Levels of Two Groups

\begin{tabular}{|c|c|c|c|}
\hline & End. Cancer group $(n=35)$ & Healthy controls $(n=40)$ & $\mathrm{p}$ value \\
\hline Age (mean, SD) & $56.80 \pm 10.19$ & $48.90 \pm 5.79$ & $0.000 *$ \\
\hline Weight (mean, SD) & $88.00 \pm 10.55$ & $79.73 \pm 7.27$ & $0.002 *$ \\
\hline BMI (mean, SD) & $34.72 \pm 4.74$ & $30.59 \pm 2.58$ & $0.001 *$ \\
\hline Gravida (mean, SD) & $3.71 \pm 2.86$ & $5.05 \pm 2.75$ & 0.085 \\
\hline Parity (mean, SD) & $3.20 \pm 2.39$ & $3.80 \pm 2.11$ & 0.384 \\
\hline Postmenapousal cases $(\mathrm{n}, \%)$ & $25(71.4 \%)$ & $20(50 \%)$ & $0.004 *$ \\
\hline Cases with diabetes $(\mathrm{n}, \%)$ & $11(31.4 \%)$ & $9(22.5 \%)$ & 0.498 \\
\hline Cases with hypertension $(\mathrm{n}, \%)$ & $20(51.7 \%)$ & $12(30 \%)$ & 0.060 \\
\hline Endometrial thickness (mean, SD) & $19.20 \pm 10.24$ & $12.11 \pm 4.25$ & $0.001 *$ \\
\hline Hemoglobin A1c levels (mean, SD) & $6.19 \pm 1.44$ & $5.61 \pm 0.58$ & $0.027 *$ \\
\hline
\end{tabular}


levels of endometrioid type versus non-endometrioid types cases $(\mathrm{p}=0.56)$. When analysing $\mathrm{HgA} 1 \mathrm{c}$ levels in diabetic versus non-diagnosed diabetics in both two groups, there is a significant difference between $\mathrm{HgA} 1 \mathrm{c}$ levels in diabetics and non-diabetics $(\mathrm{p}<0.001)$. Diabetics had a higher average $\mathrm{HgA1c}(7.29 \pm 1.59$, range 5.1-12.8) versus non-diabetics $(5.87 \pm 0.89$, range $4.4-8.6)$.

\section{Discussion}

Endometrial cancer is the most common seen gynecologic cancer in developed countries. There is established risk factors for endometrial cancer. These are hypertension, obesity, diabetes, nulliparity and anovulation. Endometrial cancer is staged surgically and have some poor prognostic factors such as advancing age, higher tumor grade, non-endometrioid histologic subtype, deeper myometrial invasion, presence of lymphovascular space invasion, amount of extrauterine spread and amplification of oncogenes, especially HER$2 /$ neu (Ludwig, 1995). It is reproted in some studies that diabetes is an indenependent risk factor for endometrial cancer (Chang et al., 2007). Also, in diabetic patients, hyperinsulinemia and impaired glucose metabolism have been hypothesized to increase the risk of many cancers, including those of the breast, genitourinary and gastrointestinal (Rinaldi et al., 2008; Tangjitgamol et al., 2010). The mechanism of tumor development in case of hyperglycemia is hypothesized as action of glucose on the production of insulin and insulin-like growth factor (IGF)1 and enhancement of tumor development by stimulating cell proliferation and inhibition of apoptosis (Travier et al., 2007). $\mathrm{HgA} 1 \mathrm{c}$ is marker for gylcemic control reflecting overall glucose levels for 3 months prior to testing.

Elevated $\mathrm{HgA1c}$ levels has been associated with increased risk of many cancers including breast, genitourinary and gastrointestinal cancers (Rinaldi et al., 2008). Siddiqui et al. (2008) reported in a case-control study that elevated $\mathrm{HgA} 1 \mathrm{c}$ is an independent predictor of aggressive clinical behavior in patients with colorectal cancer. Impaired glucoe intolerance as measured by $\mathrm{HgA} 1 \mathrm{c}$ was found to be significantly increased in endometrial cancer cases when compared to patients with other cancer (Levran et al., 1984). Travier et al. (2007) reported that women with elevated blood glucose levels have an increased risk for genital cancers and in particular endometrial cancer. As consistent with these data from literature, in our study we found that $\mathrm{HgA} 1 \mathrm{c}$ levels in endometrial cancer group was found statistically significantly higher than healthy control groups and therefore this support the hypothesis that glucose may increase the risk for cancer through its action on the production of insulin and IGF-I.

When evaluating the literature about the relationship of glycemic control to stage and grade of endometrial cancer, we have found a study done by Folsom et al. (2004) and they reported that diabetes mellitus was associated with poorer survival after endometrial cancer, independent of tumor stage and grade. Hovewer, in that study, $\mathrm{HgA} 1 \mathrm{c}$ levels or blood glucose level were not investigated as a marker. Stevens et al. (2012) reported a study investigating
$\mathrm{HgA} 1 \mathrm{c}$ levels and its relationship with stage and grade of type I endometrial cancer. They evaluated 82 type I endometrial cancer cases as higher number from our study and found that elevated preoperative $\mathrm{HgAlc}$ has a trend toward a higher stage at the time of diagnosis but this was not statistically significant. In our study consistently with this data, we found no significant difference with regards to stage and grade of tumor to HgA1c levels. We evaluated not only type I endometrial cancer also type II cancer cases were included and we found no statistically significant difference between $\mathrm{HgA} 1 \mathrm{c}$ levels of these two hystologic types.

The potential limitation of our study is that it was conducted retrospectively and the data was collected from medical records. Another weakness of our study is the small number of patients with 35 cancer cases. In our opinion, there is need of large series in order to find conclusive results about relationship between $\mathrm{HgA} 1 \mathrm{c}$ levels and cancer cases. Also, there were more patients with low-stage and low grade endometrial cancer than high grade and stage. This is thought to be because of abnormal uterine bleeding is the most common symptom and this condition prompts the patient to take medical help. It is reported in a study that $68 \%$ of endometrial cancers are diagnosed at an early stage in the USA (Smith, 2009). This data is comparable to our findings where $57 \%$ of cases were in eraly stage. Therefore the low number of late-stage cancers in general as well as in our study itself limits the ability to conclude a potential relationship between $\mathrm{HgA} 1 \mathrm{c}$ levels and tumor grade or stage.

In conclusion, poor glycemic control evaluated by serum $\mathrm{HgA}$ 1c levels seems to have an association with increased risk factor for developing endometrial cancer. This results are also consistent with previous findings that identified elevated glucose levels, leading to elevated insulin levels and related physiologic determinants, as potential mechanisms and risk factors for cancer. In this retrospective study, we did not find any significant relationship between $\mathrm{HgA} 1 \mathrm{c}$ levels and advanced stage or grade of endometrial cancer. Also, we found no significant difference between $\mathrm{HgA} 1 \mathrm{c}$ levels of two hystologic types of endometrial cancers. In order to establish a relationship between $\mathrm{HgA} 1 \mathrm{c}$ levels and endometrial cancer, studies with larger case series are needed.

\section{References}

Binesh F, Akhavan A, Behniafard N, et al (2014). Endometrial adenocarcinoma: Clinopathologic and survival characteristics in Yazd, Iran. Asian Pac J Cancer Prev, 15, 2797-801.

Chang SC, Lacey Jr JV, Brinton LA, et al (2007). Lifetime weight history and endometrial cancer risk by type of menopausal hormone use in the NIH-AARP diet and health study. Cancer Epidemiol Biomarkers Prev, 16, 723-30.

Folsom AR, Anderson KE, Sweeney C, et al (2004). Diabetes as a risk factor for death following endometrial cancer. Gynecol Oncol, 94, 740-5.

Kaaks R, Lukanova A (2001). Energy balance and cancer: the role of insulin and insulin-like growth factor-I. Proc Nutr Soc, 60, 91-106.

Lawrence C (1987). Smoking, body weight and early stage endometrial cancer. Cancer, 59, 1665-9. 


\section{Erbil Karaman et al}

LeRoith D, Roberts CT Jr (2003). The insulin-like growth factor system and cancer. Cancer Lett, 195, 127-37.

Levran D, Modan M, Menczer J, et al (1984). Increased rate of glucose intolerance in endometrial cancer-a communitybased study. Gynecol Obstet Invest, 18, 190-3.

Ludwig H (1995). Prognostic factors in endometrial cancer. Int J Gynecol Obstet, 49, 1-7.

Mahboubi E, Eyler N, Wynder EL (1982). Epidemiology of cancer of endometrium. Clin Obstet Gynecol, 302, 729-31.

Rinaldi S, Rohrmann S, Jenab M, et al (2008). Glycosylated hemoglobin and risk of colorectal cancer in men and women, the European prospective investigation into cancer and nutrition. Cancer Epidemiol Biomarkers Prev, 17, 3108-15.

Rohlfing CL, Little RR, Wiedmeyer HM, et al (2000). Use of $\mathrm{GHb}(\mathrm{HbAlc})$ in screening for undiagnosed diabetes in the U.S. population. Diabetes Care, 23, 187-91.

Siddiqui AA, Spechler SJ, Huerta S, et al (2008) Elevated HbA1c is an independent predictor of aggressive clinical behavior in patients with colorectal cancer: a case-control study. Dig Dis Sci, 53, 2486-94.

Smith RA, Cokkinides V, Brawley OW (2009). Cancer screening in the United States, 2009: a review of current American Cancer Society guidelines and issues in cancer screening. CA Cancer J Clin, 59, 27-41.

Stevens EE, Yu S, Sise VM, et al (2012). Hemoglobin A1c and the relationship to stage and grade of endometrial cancer. Arch Gynecol Obstet, 286, 1507-12.

Tangjitgamol S, Manusirivithaya S, Srijaipracharoen S, et al (2010). Clinicopathological features including hormonal receptor expression and survival in young endometrial cancer patients: a case control study. Asian Pac J Cancer Prev, 11, 1487-92.

Travier N, Jeffreys M, Brewer N, et al (2007). Association between glycosylated hemoglobin and cancer risk: a New Zealand linkage study. Ann Oncol, 18, 1414-9. 